\title{
Myocardiocyte autophagy in the context of myocardiocytes regeneration: a potential novel therapeutic strategy
}

\author{
Basheer Abdullah Marzoog ${ }^{*}$ (1) and Tatyana Ivanovna Vlasova
}

\begin{abstract}
Background: The regeneration strategy involves several aspects, such as reprogramming aspects, targeting pathophysiological processes, and inducing the physiological one. Autophagy targeting is a potential physiological/ pathogenetic strategy to enhance myocardiocytes' function. Myocardiocytes' injury-related death remains to be the highest in our era. Unfortunately, myocardiocytes have a limited proliferation capacity to compensate for what was lost by infarction. However, partially injured myocardiocytes can be preserved by improving the autophagy process of myocardiocytes.

Main text: Autophagy induction involved controlling the cellular and subcellular environment as well as gene expression. Autophagy is well known to prolong the longevity of cell and human life. Inhibition of the mTOR receptor, proapoptotic gene Bnip3, IP3, and lysosome inhibitors, inhibition of microRNA-22 and overexpression of microRNA-99a, modulators of activated protein kinase with adenosine monophosphate, resveratrol, sirtuin activators, Longevinex and calcium lowering agents can promote physiological myocardiocyte autophagy and improve postmyocardial modulation and recovery speed. The paper aimed to assess autophagy role in myocardiocytes regeneration modulation.
\end{abstract}

Conclusions: The autophagy strategy can be applied to infarcted myocardiocytes, as well as heart failure. However, cell self-eating is not the preferred therapy for preserving injured myocardiocytes or causing regeneration.

Keywords: Autophagy and regeneration, Myocardial infarction and myocardiocyte, Pathogenesis and pathophysiology, Molecular medicine

\section{Introduction}

The misery of myocardial infarction-associated death remains the most frequent despite the remarkable advances in molecular biopathophysiology. Current censuses showed that more than 4 hundred thousand individuals globally suffer from cardiovascular issues. Furthermore, the misery continues to involve approximately 18 million death annually [1].

The impaired myocardiocytes include ischemic heart, infarcted heart, and chronic heart failure. The

*Correspondence: marzug@mail.ru

National Research Mordovia State University, Bolshevitskaya Street, 68, Saransk, Rep. Mordovia, Russia 430005 pathogenesis is quite similar and involved autophagy impairment or complete cessation. Restoration of impaired myocardiocyte function remains a challenge for patients with injured myocardiocytes. Despite the current therapies to enhance antioxidant defense mechanisms (induced by autophagy) of myocardiocytes against oxidative stress, it does still not reach the required goal [2-4]. Perhaps a better intervention would be to modify the natural detoxification system that includes autophagy and an antioxidant defense system simultaneously. However, autophagy is part of the antioxidant defense system; therefore, autophagy induction probably replaces antioxidant therapy. 
Autophagy term returns to 1963 when C de Duve's firstly used this term and later discoveries continued by Yoshinori Ohsumi [5]. Induction of myocardiocytes autophagy involved regulation of gene expression, cytoplasmic and cellular environment modulations. Selfeating at hunger seems not a good idea, but for survival reasons, the cells are adjusted to degrade themselves in hope of rescue.

More recently, studies have supported the hypothesis that autophagy improved myocardiocyte regeneration through several mechanisms, including special microRNAs (microRNA-22 inhibition, MicroRNA-421 overexpression, and microRNA-99a overexpression) and the expression of damage-regulated autophagy modulator 1 (DRAM1) [6-18] (Fig. 1) DRAM1 induces autophagy through inhibition of phosphoinositide 3-kinase-AktmTOR-ribosomal protein S6 axis [19].

Targeted microRNAs can be recruited as a potential inducer for autophagy. However, several microRNAs act as autophagy inhibitors such as MIR23A, MIR29A, and MIR138-5p [20].

However, autophagy can be activated through hyperthermia and hypoxia mediated by IGF-1, food deprivation, inhibition of mTOR signaling, chaperones, and FOXO transcription factors. In addition, autophagy can be promoted through resveratrol, LY294002 (LY), metformin, and Velcade [5, 21-27]. Whereas 3-methyladenine (3-MA) and bafilomycin A1 are well-known inhibitors of autophagy [5].

Three main signaling transduction groups regulate autophagy: first includes pathways that directly involve core autophagy-related proteins (ATG) such as PI3KAKT-, AMPK-pathways, and mTOR-; second includes signaling pathways that regulate ATG proteins activity such as cAMP-, $\mathrm{Ca}^{2+}$-, and RTK/Rap1/Ras/MAPKpathways; and finally, ATG proteins transcription regulation by mainly NF-kB-, HIF-1-, TFEB, TFE3, and

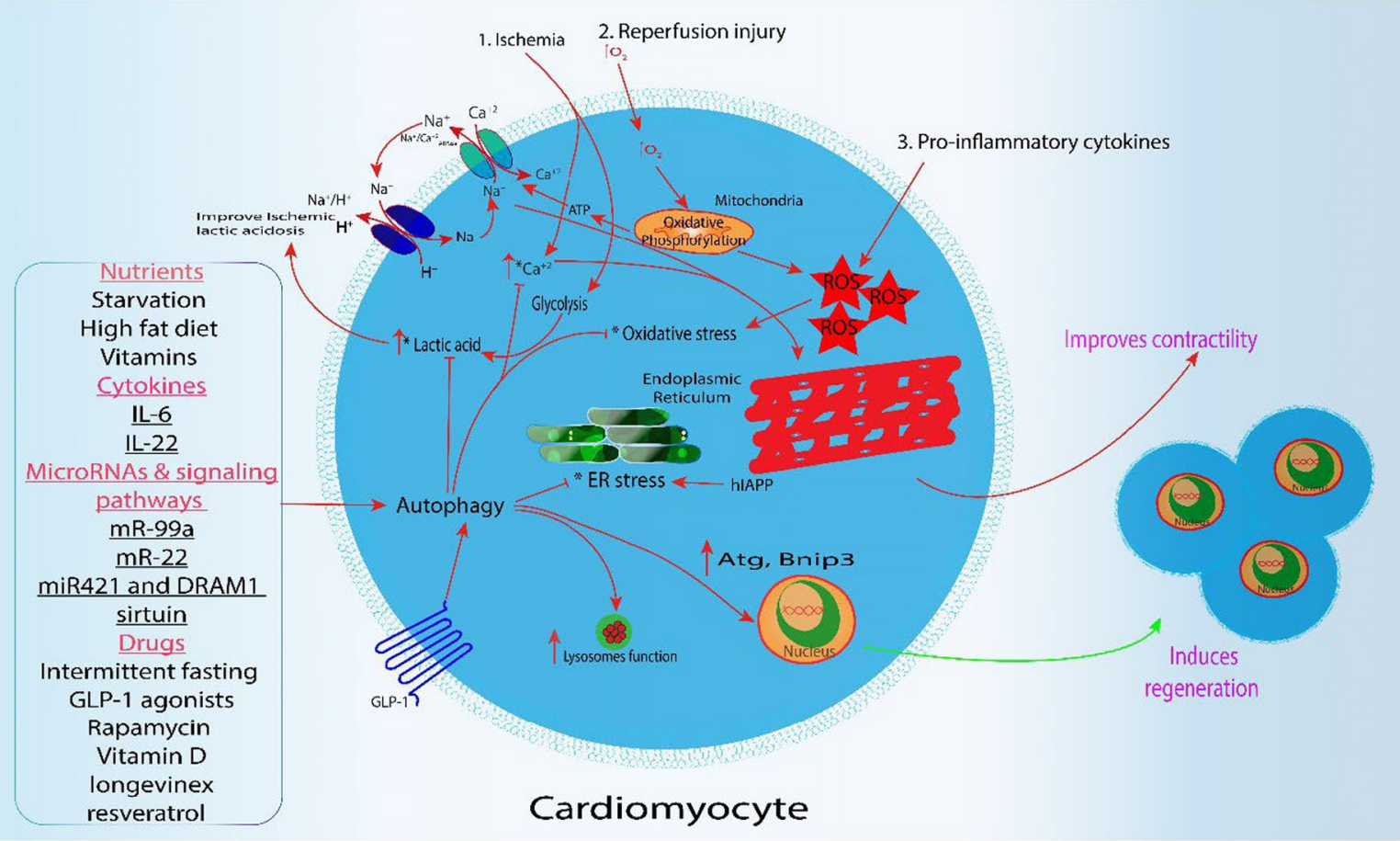

Fig. 1 Autophagy in the context of myocardiocyte regeneration and preservation of partially impaired myocardiocytes. Induction of myocardiocyte autophagy ameliorates regeneration strategies and increases myocardiocyte longevity. The regeneration and autophagy induction mechanisms involved the activation of several common genes, transcription factors, microRNAs (all the mentioned microRNAs must be overexpressed, not necessarily simultaneously), and epigenetics that are required for both processes. Autophagy activation induces the expression of Atg proteins that probably interfere with the proliferation signaling pathway and activate it. However, it remains to be elucidated since the signaling machinery is not fully understood. The role of autophagy in maintaining myocardiocytes' mass and function is through resolving oxidative stress, stress of the endoplasmic reticulum (ER), and reducing the lactic acid and calcium paradoxes (these functions indicated by asterisks) in the basal metabolite and ischemia as well as reperfusion. Subsequently, autophagy improves the intracellular homeostasis including contractile function and regeneration capacity of the myocardiocytes. GLP-1 group of anti-diabetic medications in addition to other factors can affect autophagy. Oxidative stress and endoplasmic reticulum stress are critical to maintaining autophagy and myocardiocyte mass from apoptosis and cell death, which are necessary to maintain cardiac function. IL interleukin, GLP-1 glucagon-like peptide-1, ROS reactive oxygen species 
FOXO-pathways as well as their subregulators such as NR1I2 and ESR1[28].

Therapeutic applications of autophagy are not limited to myocardiocytes but can be recruited to cure cancer, neurodegenerative diseases, infections, and promote the function of impaired cells. Autophagy impairment is a hallmark of the pathophysiology of these diseases. The self-eating of microorganisms, cancer cells, and the enhancement of impaired function of neurons and myocardiocytes make autophagy a physiological and pathological catabolic process, but its regulation can be effectively used as a therapeutic strategy.

The paper sought to assess the potential mechanism of autophagy in myocardiocyte organelles and the role of autophagy in regeneration enhancement and preserving of the partially impaired myocardiocytes.

\section{Main text}

\section{Myocardiocyte autophagy: potential mechanism}

Three well-known mechanisms are responsible for the degradation of intracellular debridements. According to the signaling markers that flag debridements, microautophagy, macroautophagy, and chaperone-mediated autophagy are present. Recent advances in molecular medicine have revealed that several autophagy-related proteins are involved in autophagy regulation, such as systems that produce modified complexes Atg8-PE and Atg5-Atg12-Atg16 [29]. Approximately 35 autophagyrelated protein (Atg) genes have been identified, 17 of which are indispensable. The molecular mechanism of myocardiocyte autophagy involved several complex signaling pathways and structures such as Beclin1-Atg14LVps15-Vps34 proteins. Phagophore is the first phase of autophagosome formation. Two primary complexes are involved in autophagosome formation, each of which has its role in norm and pathology [30, 31]. The first complex consists of III PI3K Vps34, Atg6/Beclin1, Atg14, and $V p s 15 / p 150.73$. Whereas the second complex mainly involved the serine/threonine kinase Atg1 signaling axis that its functionality depends on the presence of LC3 (microtubule-associated protein light chain 3), GATE16 (Golgi-associated ATPase enhancer of $16 \mathrm{KDa}$ ), and GABARAP (G-amino butyric acid type A receptor-associated protein) proteins [29].

The second phase of autophagy is elongation that involved ubiquitin-like conjugation pathways; Atg8/ MAP-LC3/GABAP/GATE-16 and Atg12 systems. Atg8 protein undergoes cleavage for its carboxyl-terminal by cysteine protease Atg4. Then, Atg4 undergoes a long complexed signaling cascade involving activation of Atg4 by Atg7 and Atg3 that culminate in activation of Atg8 and it is binding with the LC3 -II of the lysosome and formation of Atg8/LC3-II complex. The Atg8/LC3 -II complex bind with autophagosome in a covalent bound unless Atg4 cleaves it to be recycled and degraded by the phagolysosome [29] (Fig. 2).

The direction of autophagosome and lysosome is strictly regulated by transcription and post-transcription modulations, as well as epigenetics. Furthermore, the formation of an autolysosome is crucial and involved genetic/epigenetic/transcriptional/posttranscriptional modifications [32]. TFEB, MiT, FOXO, forkhead box, E2F1, and NF-kB transcription factors are involved in the regulation of autophagy. Epigenetics is also involved, as histone post-translational modifications include methylation, acetylation, and deacetylation.

Induction of myocardiocyte autophagy occurs physiologically under hypoxic conditions. Several mechanistic pathways are potentially possible to take place, Inhibition of mTOR receptor, proapoptotic gene Bnip3, IP3, and lysosome inhibitors depending on the hypoxia severity. During anoxia, hypoxia-inducible factor $1, \alpha$ subunit (HIF1A) is activated. HIF1A induces the expression of the Bnip3 gene (encode for BH3-only protein) which is required for disrupting the $\mathrm{BCL} 2-\mathrm{BECN} 1$ interaction by competitive binding of BCL2 (B-cell CLL/lymphoma 2) [33]. Bnip3 is an important gene for activating autophagy during hypoxic and non-hypoxic states.

\section{Autophagy on the road of organelles}

Autophagy of subcellular components is crucial for maintaining healthy cells. Furthermore, organelle product autophagy is involved in maintaining the longevity of myocardiocytes. Interestingly, autophagy not only maintains the health of cells; therefore, the idea of living longer is now combined with living healthy $[34,35]$. Organelles' autophagy involved the degradation of impaired mitochondria (mitophagy), impaired endoplasmic reticulum and peroxisomes (pexophagy), nucleus segments (micronucleophagy), ribosomes (ribophagy) [36]. The impaired organelles autophagy is considered a macroautophagy type. However, microautophagy also occurs under specific conditions that are not discussed here.

\section{Mitophagy in myocardiocytes}

Mitochondria are the dome of energy in living cells. Energy is required almost for each cellular process. The impairment of mitochondrial functions and the reduction of precursor energy production sources contribute as a primary pathogenetic clue to most pathologies, including myocardiocyte infarction [37, 38]. Mitochondrial function correction involves mitochondrial autophagy, which is an optimal therapeutic target to eliminate the sequelae of mitochondrial dysfunction (ATP deficiency, reactive oxygen species, and cell apoptosis). The first sign of mitochondrial injury 


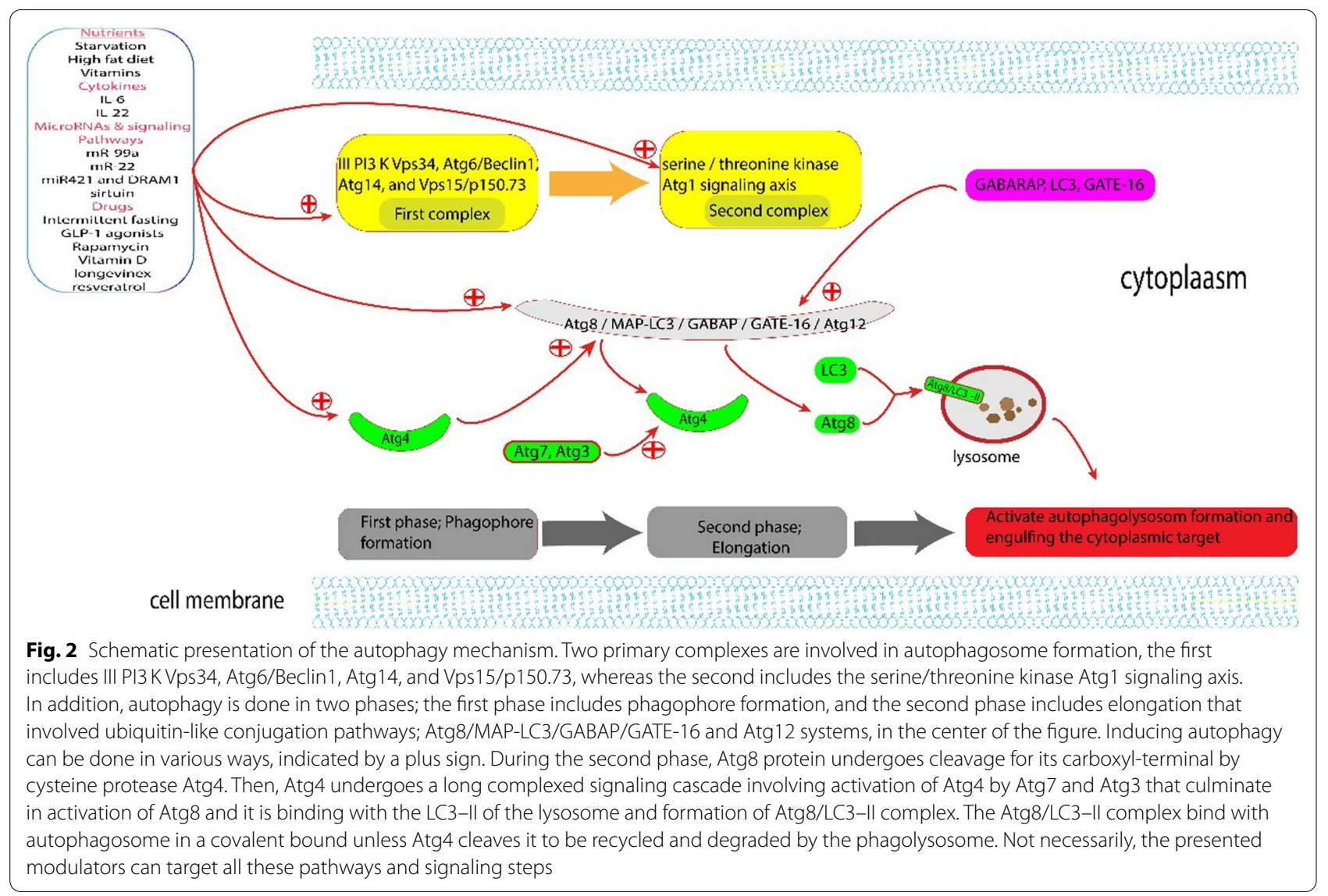

is mitochondrial DNA damage caused by free radicals and inflammatory cytokines [39]. Mitochondria, like any other organelles, are sensitive to ischemias and hypoxemias, as well as ischemic reperfusion injuries. Preserving partially injured mitochondria involves correction of physiological activity by autophagy and reduction of the production of free radicals. Furthermore, the NLRP3 inflammasome is activated during ischemia and starvation conditions [40, 41]. The complete signaling mechanism involved in the correction of mitochondrial function by autophagy is not yet fully understood and requires additional research. However, the statistical analysis of several studies concluded that giving autophagy agonists reduce the oxidative stress of healthy and ischemic myocardiocyte [23, 42].

Mitophagy and pexophagy involve Slt2 and Hog1 signal transduction pathways, each of which has its role in the pathogenesis of myocardiocytes function impairment [43]. However, in yeast MAPK signaling cascade plays a key role in determining selective autophagy [44].

\section{Autophagy of the endoplasmic reticulum of myocardiocytes}

Two stages are in front of the endoplasmic reticulum to pass the infarction; first, to preserve from ischemia and second, to rescue from ischemic reperfusion injury [45]. When the endoplasmic reticulum is in a deficit of energy, it begins to unfold proteins to produce the unfolded protein response [46]. Usually, these proteins aggregate in the cytoplasm of the myocardiocytes to form inclusion around the nucleus and cell membrane. Degradation of such pathological proteins is crucial to release the myocardiocytes from the endoplasmic reticulum stress state. Failure to degrade unfolded proteins results in activation of endoplasmic reticulum autophagy mechanisms. However, isolated autophagy of the proliferative endoplasmic reticulum can be autophagy.

The smooth endoplasmic reticulum is also involved in the pathogenesis of myocardiocyte damage during infarction. Well known, the smooth endoplasmic reticulum is a storage for cations primarily calcium. During attacks of ischemia, the calcium content of the intraendoplasmic 
reticulum will be released into the cytoplasm, leading to persistent contraction of poorly oxygenated myocardiocytes [47]. Furthermore, after reperfusion, a large amount of oxygen and calcium reenters the myocardiocyte from the extracellular space, resulting in a worse condition of calcium content and oxygen in the myocardiocyte, which consequently leads to the formation of oxygen free radicals and a large amount of intracellular calcium and subsequently, damage to the membranes of the membraned structures especially which have a high lipid ratio [48, 49].

Endoplasmic reticulum autophagy enhances the metabolic outputs of the rough endoplasmic reticulum and improves the cations controlled by the smooth endoplasmic reticulum. The signaling mechanism in which autophagy induces its effects on the endoplasmic reticulum remains an area for future research. However, a more recent study showed that the use of a highly selective agonist of $\alpha 2$-adrenergic receptors, dexmedetomidine improves myocardiocyte ischemic/reperfusion injury by down-regulation of the endoplasmic reticulum stress signaling pathway that includes glucose-regulated protein 78 (GRP78), protein kinase R-like endoplasmic reticulum kinase (PERK), homologous protein C/EBP (CHOP), inositol-requiring protein 1 (IRE1), and activating transcription factor 6 (ATF6) [50].

\section{Pexophagy in myocardiocyte}

Starvation and other unfavorable conditions (e.g., in Saccharomyces cerevisiae glucose-containing medium without a nitrogen source) induce activation of the selfeating pathophysiological mechanisms to involve peroxisomes autophagy. Selective autophagy of peroxisomes can be as micro- and macroautophagy. The mechanism involved in pexophagy includes the formation of phagophores from a particular cytosolic phagophore assembly site that later engulfs the impaired peroxisome to form an autophagosome. The molecular mechanism that initiates, directs cargo, fusion of autophagosome with lysosome to form autolysosome, and degrades cargo content (peroxisome) is identical in other organelles [29]. The phagophore assembly site, where the double membrane vacuole is synthesized, is believed to be initiated by the core autophagy-related protein ATG9L1 [51]. ATG9L1 functionality depends on Atg17, Atg29, and Atg31 proteins in addition to the dispensable Atg11 protein [52]. However, debate remains on the mechanism that involved the synthesis and the primary place of the phagophore assembly site for each organelle. Understanding of the possible mechanisms would be recruited to target organelles autophagy on the subcellular level which will have the highest drug target selectivity.

\section{Autophagy in the context of myocardiocyte regeneration} Regeneration strategy of impaired myocardiocytes involves proliferation/differentiation of already mature myocardiocytes, but unfortunately, adult myocardiocytes regenerate approximately $1 \%$ per year [53]. Therefore, a surrogate strategy is required such as preserving the partially impaired myocardiocytes through autophagy induction simultaneously with regeneration. Currently, regeneration can be through transdifferentiation of mesoderm derived cells/local cardiac stem cell into ventricular/atrial myocardiocytes through epigenetic modifications (e.g., histone methylation, acetylation, deacetylation)/cardiac transcription factors (e.g., GATA5, Mef2c, Tbx5, Nkx2.5)/small molecules (e.g., CHIR99021, A83-0,1BIX01294, AS8351, Y27632, OAC2, SU16F, SC1, JNJ10198409)/cardiac non-coding microRNAs (e.g., miR15, miR-128, miR-101a, and miR-133). Also regeneration can be through differentiation of induced/pluripotent stem cells from various origins such as cardiac or skin fibroblast by Yamanaka factors (Oct4, Sox2, Klf4, C-myc) into myocardiocytes via cardiac transcription factors (e.g., GATA5, Mef2c, Tbx5, Nkx2.5) and via special microenvironment [53].

Our hypothesis of autophagy-based myocardiocyte regeneration endorses the cross-activation of a specific common mechanism between autophagy and myocardiocyte regeneration (for example, by transcription factors). For better understanding, carrying research on special cardiac microRNAs, cardiac transcription factors, and epigenetics is required, which is our current project. However, it remains difficult to control for other factors' role in the regeneration strategy such as oxidative stress and endoplasmic stress which each of them plays a key role in the pathophysiology and regeneration aspects of myocardiocytes. Each of these pathophysiological events required extra investigation to determine their role in regeneration that accordingly, we may regulate their activity.

In vitro, more recent findings addressed leptin $b$ an important role in myocardiocytes proliferation capacity after controlled myocardiocyte injury [54].

\section{Currently used medications in the context of autophagy enhancement}

Unfortunately, currently, the use of drugs to induce autophagy is still a poorly developed concept. However, several medications that are used for the treatment of several other common diseases have been identified to induce autophagy of cells of the whole organism without organelle selectivity. Approximately 225 autophagy modulators have been identified, of which 174 are autophagy inducers, 31 are suppressors, while 20 have dual effects 
Table 1 Group of medications with potentiality of autophagy enhancement, not limited to this list

\begin{tabular}{|c|c|c|}
\hline Medication & Target & Potential mechanism \\
\hline Resveratrol & AMPK, mTOR, a serine/threonine kinase complex & $\begin{array}{l}\text { Activation of AMPK, H3K79 methylation, inhibits mTOR; enhances the interaction } \\
\text { between mTOR and DEPTOR (DEP-domain containing mTOR-interacting protein) }\end{array}$ \\
\hline Longevinex & Pl-3-kinase-Akt pathway & $\begin{array}{l}\text { Activation of Pl-3-kinase-Akt pathway and enhance expression of mitochondrial Sirt1 } \\
\text { and Sirt3 }\end{array}$ \\
\hline Artenimol & Mitogen-activated protein kinase (MAPK) & induce MAPK activity; transition metals copper and zinc intracellularly \\
\hline Metformin & AMPK, mammalian target of rapamycin (mTOR) & Activation of AMPK, inhibits mTOR, S6 kinase and ULK1 phosphorylation \\
\hline Velcade & Chymotrypsin-like activity of the proteasome & reversible inhibitor of the chymotrypsin-like activity of the proteasome \\
\hline Rapamycin & mTOR & mTOR inhibitor \\
\hline Sirtuin & FOXO-dependent pathway & Enhance $\mathrm{NAD}^{+}$production \\
\hline
\end{tabular}

depending on the biological context (e.g., melatonin in normal cells induces autophagy, but in tumor cells reduces autophagy) [55] (Table 1) Autophagy modulators have more than 1005 targets, every modulator has multiple targets and usually targets not the autophagy proteins directly but their regulators.

Metformin is the most commonly used medication to manage type 2 diabetes [56]. Metformin was found to induce beta-cell insulin production as well as reduce the resistance of insulin-sensitive cells to insulin. Moreover, recent findings suggested that metformin is involved in inducing autophagy as well ferroptosis [57]. The molecular mechanism involved activation of the AMPK-MTOR/ PGC-1alpha signaling pathway [58]. Probably, activation of apoptosis genes by metformin is a protective mechanism for cells from transdifferentiation to another cell linage such as fibroblast (e.g., in post-myocardial infarction modulation scar tissue) [15].

More recently, in vivo findings have suggested that metformin administration after myocardial infarction increases heart wall rupture incidence [58].

\section{Discussion}

Autophagy is the only mechanism that can degrade long-lived proteins and organelles, unlike the ubiquitin proteasome system. Since autophagy is involved in the regulation of cell differentiation, it is likely that it can be recruited to optimize current myocardiocyte regeneration strategies, such as transcription factors in pluripotent stem cell differentiation. However, the regeneration mechanism remains in the womb stage and requires additional elucidation. But gene expression analysis demonstrated that knockdown Atg5 protein, which is involved in the autophagy regulation, results in a reduction of myocardiocytes regeneration and later fibrosis and hypertrophy as well as low ejection fraction. Regeneration of myocardiocytes involved proliferation of neo cells and angiogenesis. In part, these processes are regulated by the same genetic, epigenetic, transcription factors and non-coding microRNAs that regulate autophagy [62]. Therefore, experimental studies have found an increase in myocardiocytes regeneration during induction of autophagy [63].

Cell differentiation and proliferation are partially regulated by common molecular pathways that involved crosstalk between autophagy and Wnt catenin signaling pathways. Ischemic conditions induced autophagy of the $\beta$-catenin and Disheveled proteins by the LC3 component of autophagy. Not only that, but also the GSK3 $\beta$ signaling pathway, another part of the Wnt catenin signaling pathways, can induce autophagy by phosphorylating the TSC complex [64]. Markers of successful myocardiocyte autophagy include LC3-I/II and Beclin1 levels [65].

Interestingly, recent findings by $\mathrm{Cui}$ et al. have addressed the crucial role of lysosomal membrane permeability (LMP) in the regulation of cell death. The study demonstrated that overexpression of lysosomal membrane-associated protein 2 (Lamp2) results in induction of autophagy flow and reduction of myocardiocyte death from ischemia [66]. Transinfection of glucose, oxygen, and serum-deprived myocardiocytes with an adenovirus vector containing the Lamp2 gene resulted in a remarkable improvement in deprived cell autophagy and subsequently preserved ischemic myocardiocytes. At the molecular level, Lamp2 induces its effect through upregulation of cathepsin B and cathepsin D [67-69]. Dysregulation of Lamp2 protein results in a rare X-linked cardiomyopathy disease called Danon disease [70].

\section{Current challenges and future prospects}

So far, autophagy is well-known inducer for cellular antioxidant defense system which is necessary for protection against myocardial ischemic attacks/reperfusion injury [59]. However, recent bio-molecular advances didn't explain the potential role of autophagy in heart regeneration and especially myocardiocytes [60]. We suggest that molecular and cellular processes of autophagy involve the activation of a common signaling pathway 
for both autophagy and regeneration. Therefore, during unfavorable conditions such as low myocardiocytes perfusion (ischemia) and hypoxia, autophagy is activated which is probably also required for successful regeneration strategy.

We also suggest that an optimal level of autophagy is required for successful heart regeneration in all potential regeneration strategies. Transcriptionally, autophagy is regulated by MITF and FOXO, as the transcription families well as CREB and ATF transcription families [32, 61]. This gives a better opportunity for overregulation to target autophagy, enhance or suppress autophagy.

\section{Conclusions}

Autophagy is a natural subcellular catabolic detoxification process that involves the degradation of altered organelles and cytoplasmic components, such as proteins, to maintain cell homeostasis [71]. Autophagyinduced cell physiology can be applied to mechanically healthy and injured cells. Maintaining a high level of autophagy involved epigenetic regulation of autophagy gene expression. And with age, these epigenetic markers are lost. Therefore, cells age and impair function, including myocardiocytes. Suggesting, myocardiocytes death is primarily due to autophagy mechanisms dysregulation. Probably, a combination of reperfusion of myocardiocytes and inducing autophagy optimizes current therapy for myocardial infarction. Since autophagy reduces oxidative stress, lactic acidosis, and $\mathrm{Ca}^{+2}$ paradoxes (reperfusion injury).

Autophagy plays a key role in the term of myocardiocytes' pathogenesis and dysfunction. The basal myocardiocytes' metabolic state requires autophagy to catabolize the toxic end-products of intracellular reactions. Autophagy behavior regulation is critical in health and disease since its involvement in the basal metabolic state and pathogenesis of diseases. Therefore, induction of autophagy through a safe and effective strategy is a potential cure for myocardiocytes dysfunction.

\footnotetext{
Abbreviations

3-MA: 3-Methyladenine; LY: LY294002; GABARAP: G-amino butyric acid type A receptor-associated protein; GATE-16: Golgi-associated ATPase enhancer of 16 KDa; LC3: Microtubule-associated protein light chain 3; DRAM1: Damageregulated autophagy modulator 1; HIF1A: Hypoxia inducible factor 1, a subunit.
}

\section{Acknowledgements}

My thanks are dedicated to my love, supervisor, and professor, Tatyana Ivanovna Vlasova, for her endless patience, wisdom, insight, and support without which my work would not have been possible.

\section{Authors' contributions}

$\mathrm{MB}$ is the writer, researcher, collected and analyzed data, and revised the manuscript, while VT revised the final version. All authors have read and approved the final manuscript.
Funding

Not applicable (This research did not receive any specific grant from funding agencies in the public, commercial, or not-for-profit sectors).

Availability of data and materials

Not applicable.

\section{Declarations}

Ethics approval and consent to participate

Not applicable.

Consent for publication

Not applicable.

Competing interests

The authors declare that they have no competing interests regarding publication.

Received: 5 September 2021 Accepted: 28 December 2021

Published online: 03 March 2022

References

1. Virani SS, Alonso A, Aparicio HJ, Benjamin EJ, Bittencourt MS, Callaway CW, Carson AP, Chamberlain AM, Cheng S, Delling FN et al (2021) Heart disease and stroke statistics - 2021 update. Circulation 143(8):e254e743. https://doi.org/10.1161/CIR.0000000000000950

2. Madole MB, Bachewar NP, Aiyar CM (2015) Study of oxidants and antioxidants in patients of acute myocardial infarction. Adv Biomed Res 4:241. https://doi.org/10.4103/2277-9175.168608

3. González-Montero J, Brito R, Gajardo Al, Rodrigo R (2018) Myocardial reperfusion injury and oxidative stress: therapeutic opportunities. World J Cardiol 10(9):74-86. https://doi.org/10.4330/wjc.v10.i9.74

4. Lin Z, Jiang Y, Yang P, Sun L, Lu D (2020) A frog antioxidant peptide protects against myocardial ischemia reperfusion injury in rats. All Life 13(1):45-53. https://doi.org/10.1080/21553769.2019.1699171

5. Ohsumi $Y$ (2014) Historical landmarks of autophagy research. Cell Res 24(1):9-23. https://doi.org/10.1038/cr.2013.169

6. Li Q, Xie J, Li R, Shi J, Sun J, Gu R, Ding L, Wang L, Xu B (2014) Overexpression of MicroRNA-99a attenuates heart remodelling and improves cardiac performance after myocardial infarction. J Cell Mol Med 18(5):919-928. https://doi.org/10.1111/JCMM.12242

7. Wang J, Qian H-L, Chen S-Y, Huang W-P, Huang D-N, Hao H-Y, Ren K-F, Wang Y-B, Fu G-S, Ji J (2021) MiR-22 eluting cardiovascular stent based on a self-healable spongy coating inhibits in-stent restenosis. Bioact Mater 6(12):4686-4696

8. Cong BH, Zhu XY, Ni X (2017) The roles of microRNA-22 in myocardial infarction. Sheng Li Xue Bao 69(5):571-578

9. Xiao Y, Zhang Y, Chen Y, Li J, Zhang Z, Sun Y, Shen H, Zhao Z, Huang $Z$, Zhang $W$ et al (2019) Inhibition of microRNA-9-5p protects against cardiac remodeling following myocardial infarction in mice. Hum Gene Ther 30(3):286-301. https://doi.org/10.1089/HUM.2018.059

10. Sciarretta S, De Falco E, Frati G, Sadoshima J (2017) How to be young at heart? MiR-22 as a potential therapeutic target to boost autophagy and protect the old myocardium. Ann Transl Med 1:1

11. Wu X, Qin Y, Zhu X, Liu D, Chen F, Xu S, Zheng D, Zhou Y, Luo J (2018) Increased expression of DRAM1 confers myocardial protection against ischemia via restoring autophagy flux. J Mol Cell Cardiol 124:70-82. https://doi.org/10.1016/J.YJMCC.2018.08.018

12. Matkovich SJ, Dorn GW (2016) Feed my heart or eat it: MiR-22 decides*. J Am Coll Cardiol 68(14):1572-1574. https://doi.org/10.1016/J.JACC.2016. 07.740

13. Yang F, Qin Y, Wang Y, Meng S, Xian H, Che H, Lv J, Li Y, Yu Y, Bai Y et al (2019) Metformin inhibits the NLRP3 inflammasome via AMPK/MTORdependent effects in diabetic cardiomyopathy. Int J Biol Sci 15(5):10101019. https://doi.org/10.7150/IJBS.29680

14. Hesen NA, Riksen NP, Aalders B, Ritskes-Hoitinga M, El Messaoudi S, Wever KE (2017) A systematic review and meta-analysis of the protective 
effects of metformin in experimental myocardial infarction. PLOS ONE 12(8):e183664. https://doi.org/10.1371/journal.pone.0183664

15. Fei Q, Ma H, Zou J, Wang W, Zhu L, Deng H, Meng M, Tan S, Zhang H, Xiao $X$ et al (2020) Metformin Protects against ischaemic myocardial injury by alleviating autophagy-ROS-NLRP3-mediated inflammatory response in macrophages. J Mol Cell Cardiol 145:1-13

16. Ren J, Zhang Y (2018) Targeting autophagy in aging and aging-related cardiovascular diseases. Trends Pharmacol Sci 39:1064-1076

17. Kowara M, Borodzicz-Jazdzyk S, Rybak K, Kubik M, Cudnoch-Jedrzejewska A (2021) Therapies targeted at non-coding RNAs in prevention and limitation of myocardial infarction and subsequent cardiac remodelingcurrent experience and perspectives. Int J Mol Sci 22(11):5718. https:// doi.org/10.3390/ijms22115718

18. Guo L-L, Guo M, Yao J, Weng Y, Zhang X (2020) MicroRNA-421 improves ischemia/reperfusion injury via regulation toll-like receptor 4 pathway. J Int Med Res 48(3):300060519871863. https://doi.org/10.1177/03000 60519871863

19. LuT, Zhu Z, Wu J, She H, Han R, Xu H, Qin Z-H (2019) DRAM1 regulates autophagy and cell proliferation via inhibition of the phosphoinositide 3-kinase-Akt-MTOR-ribosomal protein s6 pathway. Cell Commun Signal 17(1):28. https://doi.org/10.1186/s12964-019-0341-7

20. Akkoc $Y$, Gozuacik D (2020) MicroRNAs as major regulators of the autophagy pathway. Biochim Biophys Acta Mol Cell Res 1867(5):118662. https://doi.org/10.1016/j.bbamcr.2020.118662

21. Wu J, Li X, Zhu G, Zhang Y, He M, Zhang J (2016) The role of resveratrolinduced mitophagy/autophagy in peritoneal mesothelial cells inflammatory injury via NLRP3 inflammasome activation triggered by mitochondrial ROS. Exp Cell Res 341:42-53

22. Bromage DI, Godec TR, Pujades-Rodriguez M, Gonzalez-Izquierdo A Denaxas S, Hemingway H, Yellon DM (2019) Metformin use and cardiovascular outcomes after acute myocardial infarction in patients with type 2 diabetes: a cohort study. Cardiovasc Diabetol 18(1):168. https://doi.org/ 10.1186/s12933-019-0972-4

23. Elmadhun NY, Sabe AA, Lassaletta AD, Chu LM, Sellke FW (2014) Metformin mitigates apoptosis in ischemic myocardium. J Surg Res 192(1):50-58. https://doi.org/10.1016/j.jss.2014.05.005

24. Luo F, Das A, Chen J, Wu P, Li X, Fang Z (2019) Metformin in patients with and without diabetes: a paradigm shift in cardiovascular disease management. Cardiovasc Diabetol 18(1):54. https://doi.org/10.1186/ s12933-019-0860-y

25. Basnet S, Kozikowski A, Makaryus AN, Pekmezaris R, Zeltser R, Akerman M, Lesser M, Wolf-Klein G (2015) Metformin and myocardial injury in patients with diabetes and ST-segment elevation myocardial infarction: a propensity score matched analysis. J Am Heart Assoc 4(10):e2314. https:// doi.org/10.1161/JAHA.115.002314

26. Calvert JW, Gundewar S, Jha S, Greer JJM, Bestermann WH, Tian R, Lefer DJ (2008) Acute metformin therapy confers cardioprotection against myocardial infarction via AMPK-ENOS-mediated signaling. Diabetes 57(3):696-705. https://doi.org/10.2337/db07-1098

27. Alfaro IE, Albornoz A, Molina A, Moreno J, Cordero K, Criollo A, Budini M (2019) Chaperone mediated autophagy in the crosstalk of neurodegenerative diseases and metabolic disorders. Front Endocrinol (Lausanne). https://doi.org/10.3389/FENDO.2018.00778/FULL

28. Ren J, Zhang Y (2018) Targeting autophagy in aging and aging-related cardiovascular diseases. Trends Pharmacol Sci 39(12):1064. https://doi. org/10.1016/J.TIPS.2018.10.005

29. Suzuki K, Ohsumi Y (2007) Molecular machinery of autophagosome formation in yeast, Saccharomyces Cerevisiae. FEBS Lett 581(11):2156-2161. https://doi.org/10.1016/J.FEBSLET.2007.01.096

30. Dikic I, Elazar Z (2018) Mechanism and medical implications of mammalian autophagy. Nat Rev Mol Cell Biol 19(6):349-364. https://doi.org/ 10.1038/S41580-018-0003-4

31. Gatica D, Chiong M, Lavandero S, Klionsky DJ (2015) Molecular mechanisms of autophagy in the cardiovascular system. Circ Res 116(3):456467. https://doi.org/10.1161/CIRCRESAHA.114.303788

32. Di Malta C, Cinque L, Settembre C (2019) Transcriptional regulation of autophagy: mechanisms and diseases. Front Cell Dev Biol. https://doi. org/10.3389/FCELL.2019.00114/BIBTEX

33. Hale AN, Ledbetter DJ, Gawriluk TR, Rucker EB III (2013) Autophagy: Regulation and role in development. Autophagy 9(7):951-972. https://doi.org/ 10.4161/auto.24273
34. Marin-Aguilar F, Lechuga-Vieco AV, Alcocer-Gomez E, Castejon-Vega B, Lucas J, Garrido C et al (2020) NLRP3 inflammasome suppression improves longevity and prevents cardiac aging in male mice. Aging Cell 19:e13050

35. Dai J, Zhang X, Li L, Chen H, Chai Y (2017) Autophagy inhibition contributes to ROS-producing NLRP3-dependent inflammasome activation and cytokine secretion in high glucose-induced macrophages. Cell Physiol Biochem 43:247-256

36. Manjithaya R, Nazarko TY, Farré JC, Subramani Suresh S (2010) Molecular mechanism and physiological role of pexophagy. FEBS Lett 584(7):13671373. https://doi.org/10.1016/J.FEBSLET.2010.01.019

37. Zhou H, Zhu P, Wang J, Zhu H, Ren J, Chen Y (2018) Pathogenesis of cardiac ischemia reperfusion injury is associated with CK2a-disturbed mitochondrial homeostasis via suppression of FUNDC1-related mitophagy. Cell Death Differ 25(6):1080-1093. https://doi.org/10.1038/ s41418-018-0086-7

38. Yang M, Linn BS, Zhang Y, Ren J (2019) Mitophagy and mitochondrial integrity in cardiac ischemia-reperfusion injury. Biochim Biophys Acta Mol Basis Dis 1865(9):2293-2302. https://doi.org/10.1016/j.bbadis.2019.05.007

39. Qin C, Gu J, Liu R, Xu F, Qian H, He Q, Meng W (2017) Release of mitochondrial DNA correlates with peak inflammatory cytokines in patients with acute myocardial infarction. Anatol J Cardiol 17(3):224-228. https:// doi.org/10.14744/AnatolJCardiol.2016.7209

40. Shimada K, Crother TR, Karlin J, Dagvadorj J, Chiba N, Chen S, Ramanujan VK, Wolf AJ, Vergnes L, Ojcius DM et al (2012) Oxidized mitochondrial DNA activates the NLRP3 inflammasome during apoptosis. Immunity 36(3):401-414. https://doi.org/10.1016/j.immuni.2012.01.009

41. Toldo S, Abbate A (2018) The NLRP3 inflammasome in acute myocardial infarction. Nat Rev Cardiol 15:203-214

42. Soraya H, Rameshrad M, Mokarizadeh A, Garjani A (2015) Metformin attenuates myocardial remodeling and neutrophil recruitment after myocardial infarction in rat. Biolmpacts 5(1):3-8. https://doi.org/10.15171/bi. 2015.02

43. Aoki Y, Kanki T, Hirota Y, Kurihara Y, Saigusa T, Uchiumi T, Kang D (2011) Phosphorylation of serine 114 on Atg32 mediates mitophagy. Mol Biol Cell 22(17):3206-3217. https://doi.org/10.1091/MBC.E11-02-0145

44. Manjithaya R, Jain S, Farré JC, Subramani S (2010) A yeast MAPK cascade regulates pexophagy but not other autophagy pathways. J Cell Biol 189(2):303-310. https://doi.org/10.1083/JCB.200909154

45. Ruan Y, Zeng J, Jin Q, Chu M, Ji K, Wang Z, Li L (2020) Endoplasmic reticulum stress serves an important role in cardiac ischemia/reperfusion injury (review). Exp Ther Med 20(6):1-1. https://doi.org/10.3892/etm.2020.9398

46. Thorp EB (2012) The myocardial unfolded protein response during ischemic cardiovascular disease. Biochem Res Int 2012:1-7. https://doi. org/10.1155/2012/583170

47. Ren J, Bi Y, Sowers JR, Hetz C, Zhang Y (2021) Endoplasmic reticulum stress and unfolded protein response in cardiovascular diseases. Nat Rev Cardiol 18(7):499-521. https://doi.org/10.1038/s41569-021-00511-w

48. Marzoog BA, Vlasova TI (2021) Membrane lipids under norm and pathology. Eur J Clin Exp Med 19(1):59-75. https://doi.org/10.15584/ejcem. 2021.1.9

49. Yan B, Liu S, Li X, Zhong Y, Tong F, Yang S (2019) Preconditioning with endoplasmic reticulum stress alleviated heart ischemia/reperfusion injury via modulating IRE1/ATF6/RACK1/PERK and PGC-1a in diabetes mellitus. Biomed Pharmacother 118:109407. https://doi.org/10.1016/j.biopha. 2019.109407

50. Yang $Y$, Wang $H$, Song $N$, Jiang $Y$, Zhang J, Meng $X$, Feng $X$, Liu H, Peng $K$, Ji F (2021) Dexmedetomidine attenuates ischemia/reperfusion-induced myocardial inflammation and apoptosis through inhibiting endoplasmic reticulum stress signaling. J Inflamm Res 14:1217-1233. https://doi.org/ $10.2147 / J$ IR.S292263

51. Mari M, Griffith J, Rieter E, Krishnappa L, Klionsky DJ, Reggiori F (2010) An Atg9-containing compartment that functions in the early steps of autophagosome biogenesis. J Cell Biol 190(6):1005-1022. https://doi.org/ 10.1083/JCB.200912089

52. Kawamata T, Kamada Y, Kabeya Y, Sekito T, Ohsumi Y (2008) Organization of the pre-autophagosomal structure responsible for autophagosome formation. Mol Biol Cell 19(5):2039-2050. https://doi.org/10.1091/MBC. E07-10-1048 
53. Marzoog BA, Vlasova TI (2021) Transcription factors are the heart of heart regeneration; A potential novel therapeutic strategy. Curr Mol Med. https://doi.org/10.2174/1566524021666210712144638

54. Kang J, Hu J, Karra R, Dickson AL, Tornini VA, Nachtrab G, Gemberling M, Goldman JA, Black BL, Poss KD (2016) Modulation of tissue repair by regeneration enhancer elements. Nature 532(7598):201-206. https://doi. org/10.1038/NATURE17644

55. Shi Q, Pei F, Silverman GA, Pak SC, Perlmutter DH, Liu B, Bahar I (2020) Mechanisms of action of autophagy modulators dissected by quantitative systems pharmacology analysis. Int J Mol Sci 21(8):2855. https://doi. org/10.3390/ijms21082855

56. Marzoog B (2021) Lipid behavior in metabolic syndrome pathophysiology. Curr Diabetes Rev. https://doi.org/10.2174/15733998176662109151 01321

57. Yang J, Zhou Y, Xie S, Wang J, Li Z, Chen L, Mao M, Chen C, Huang A, Chen $Y$ et al (2021) Metformin induces ferroptosis by inhibiting UFMylation of SLC7A11 in breast cancer. J Exp Clin Cancer Res 40(1):206. https://doi.org/ 10.1186/s13046-021-02012-7

58. Hua J, Liu Z, Liu Z, An D, Lai W, Zhan Q et al (2018) Metformin increases cardiac rupture after myocardial infarction via the AMPK-MTOR/PGC1 alpha signaling pathway in rats with acute myocardial infarction. Med Sci Monit 24:6989-7000

59. Aurora AB, Porrello ER, Tan W, Mahmoud Al, Hill JA, Bassel-Duby R, Sadek HA, Olson EN (2014) Macrophages are required for neonatal heart regeneration. J Clin Investig 124(3):1382-1392. https://doi.org/10.1172/JCl72 181

60. Lu J, Pan SS, Wang QT, Yuan Y (2018) Alterations of cardiac KATP channels and autophagy contribute in the late cardioprotective phase of exercise preconditioning. Int Heart J 59(5):1106-1115. https://doi.org/10.1536//HJ. 17-003

61. Füllgrabe J, Klionsky DJ, Joseph B (2014) The return of the nucleus: transcriptional and epigenetic control of autophagy. Nat Rev Mol Cell Biol 15(1):65-74. https://doi.org/10.1038/NRM3716

62. Gupta SK, Foinquinos A, Thum S, Remke J, Zimmer K, Bauters C, de Groote P, Boon RA, de Windt LJ, Preissl S, Hein L, Batkai S, Pinet F, Thum T (2016) Preclinical development of a microRNA-based therapy for elderly patients with myocardial infarction. J Am Coll Cardiol 68(14):1557-1571. https:// doi.org/10.1016/j.jacc.2016.07.739

63. Chávez MN, Morales RA, López-Crisosto C, Roa JC, Allende ML, Lavandero S (2020) Autophagy activation in zebrafish heart regeneration. Sci Rep 10(1):2191. https://doi.org/10.1038/s41598-020-59106-z

64. Lorzadeh S, Kohan L, Ghavami S, Azarpira N (2021) Autophagy and the Wnt signaling pathway: a focus on Wnt/ß-catenin signaling. Biochim Biophys Acta Mol Cell Res 1868(3):1 18926. https://doi.org/10.1016/j.bbamcr. 2020.118926

65. Mukherjee S, Ray D, Lekli I, Bak I, Tosaki A, Das DK (2010) Effects of Longevinex (modified resveratrol) on cardioprotection and its mechanisms of action. Can J Physiol Pharmacol 88(11):1017-1025. https://doi.org/10. 1139/Y10-082/ASSET/IMAGES/LARGE/Y10-082F7.JPEG

66. Cui L, Zhao L-PP, Ye J-YY, Yang L, Huang YY, Jiang X-PP, Zhang Q, Jia J-ZZ, Zhang D-XX, Huang YY (2020) The lysosomal membrane protein Lamp2 alleviates lysosomal cell death by promoting autophagic flux in ischemic cardiomyocytes. Front Cell Dev Biol 8:31

67. Law CY, Siu CW, Fan K, Lai WH, Au KW, Lau YM, Wong LY, Ho JCY, Lee Y, Tse HF et al (2016) Lysosomal membrane permeabilization is involved in oxidative stress-induced apoptotic cell death in LAMP2-deficient IPSCs-derived cerebral cortical neurons. Biochem Biophys Rep 5:335-345. https://doi.org/10.1016/J.BBREP.2016.01.010

68. Gómez-Sintes R, Ledesma MD, Boya P (2016) Lysosomal cell death mechanisms in aging. Ageing Res Rev 32:150-168. https://doi.org/10. 1016/J.ARR.2016.02.009

69. Repnik U, Česen MH, Turk B (2016) Measuring cysteine cathepsin activity to detect lysosomal membrane permeabilization. Cold Spring Harb Protoc 2016(5):453-458. https://doi.org/10.1101/PDB.PROT087114

70. Rowland TJ, Sweet ME, Mestroni L, Taylor MRG (2016) Danon diseasedysregulation of autophagy in a multisystem disorder with cardiomyopathy. J Cell Sci 129(11):2135-2143. https://doi.org/10.1242/jcs.184770

71. Galati S, Boni C, Gerra MC, Lazzaretti M, Buschini A (2019) Autophagy: a player in response to oxidative stress and DNA damage. Oxid Med Cell Longev 2019(4):1-12. https://doi.org/10.1155/2019/5692958

\section{Publisher's Note}

Springer Nature remains neutral with regard to jurisdictional claims in published maps and institutional affiliations.

\section{Submit your manuscript to a SpringerOpen ${ }^{\circ}$ journal and benefit from:}

- Convenient online submission

- Rigorous peer review

- Open access: articles freely available online

- High visibility within the field

- Retaining the copyright to your article

Submit your next manuscript at $\boldsymbol{\Delta}$ springeropen.com 